University of Nebraska - Lincoln

DigitalCommons@University of Nebraska - Lincoln

6-1-2008

\title{
Local probing of relaxation time distributions in ferroelectric polymer nanomesas: Time-resolved piezoresponse force spectroscopy and spectroscopic imaging
}

\author{
Brian J. Rodriguez \\ USA \\ Stephen Jesse \\ Oak Ridge National Laboratory, sjesse@ornl.gov \\ Jihee Kim \\ University of Nebraska - Lincoln \\ Stephen Ducharme \\ University of Nebraska, sducharme1@unl.edu \\ Sergei V. Kalinin \\ Oak Ridge National Laboratory, sergei2@ornl.gov
}

Materials Science and Technology Division, Oak Ridge National Laboratory, Oak Ridge,Tennessee 37831,

Follow this and additional works at: https://digitalcommons.unl.edu/physicsducharme

Part of the Physics Commons

Rodriguez, Brian J.; Jesse, Stephen; Kim, Jihee; Ducharme, Stephen; and Kalinin, Sergei V., "Local probing of relaxation time distributions in ferroelectric polymer nanomesas: Time-resolved piezoresponse force spectroscopy and spectroscopic imaging" (2008). Stephen Ducharme Publications. 38.

https://digitalcommons.unl.edu/physicsducharme/38

This Article is brought to you for free and open access by the Research Papers in Physics and Astronomy at DigitalCommons@University of Nebraska - Lincoln. It has been accepted for inclusion in Stephen Ducharme Publications by an authorized administrator of DigitalCommons@University of Nebraska - Lincoln. 


\title{
Local probing of relaxation time distributions in ferroelectric polymer nanomesas: Time-resolved piezoresponse force spectroscopy and spectroscopic imaging
}

\author{
Brian J. Rodriguez, ${ }^{1,2, a)}$ Stephen Jesse, ${ }^{2}$ Jihee Kim, ${ }^{3}$ Stephen Ducharme, ${ }^{3}$ and \\ Sergei V. Kalinin ${ }^{1,2, b)}$ \\ ${ }^{1}$ Materials Science and Technology Division, Oak Ridge National Laboratory, Oak Ridge, \\ Tennessee 37831, USA \\ ${ }^{2}$ Center for Nanophase Materials Sciences, Oak Ridge National Laboratory, Oak Ridge, \\ Tennessee 37831, USA \\ ${ }^{3}$ Department of Physics and Astronomy and Nebraska Center for Materials and Nanoscience, \\ University of Nebraska, Lincoln, Nebraska 68588-0111, USA
}

(Received 10 April 2008; accepted 19 May 2008; published online 11 June 2008)

\begin{abstract}
Time-resolved piezoresponse force spectroscopy (TR-PFS) and spectroscopic imaging are developed to probe the spatial variability of relaxation behavior in nanoscale ferroelectric materials and structures. TR-PFS was applied to study polarization dynamics in polyvinylidine fluoride and trifluoroethylene nanomesas. We demonstrate that polarization relaxation in ferroelectric polymers is slow even on the $\sim 10 \mathrm{~nm}$ length scale of piezoresponse force microscopy (PFM) signal generation. Furthermore, the relaxation times are found to be nonuniform within the nanomesa, indicative of a complex internal structure. The applicability of TR-PFM for studies of polarization dynamics in ferroelectric polymers and relaxors is discussed. (C) 2008 American Institute of Physics. [DOI: $10.1063 / 1.2942390]$
\end{abstract}

Polyvinylidene fluoride (PVDF) and its copolymers are prototypical examples of electroactive ferroelectric polymers that have long attracted attention for applications in transducers, sensors, and actuators. ${ }^{1}$ The recent advances in Langmuir-Blodgett (LB) deposition techniques have allowed fabrication of high-quality nanometer-thick films of VDF copolymers. The high surface stability of PVDF has allowed integration with nanowires and nanotubes in ferroelectricgate field effect transistors for nonvolatile data storage device applications, suggesting the potential of polymer-based nonvolatile electronic devices.

One of the crucial issues in ferroelectric-based devices is the polarization switching time. Unlike ferroelectric perovskites that typically switch on a nanosecond time scale, macroscopic studies have suggested that switching processes in PVDF are relatively slow and polarization switching on the time scales from $10^{-3}$ to $10^{2} \mathrm{~s}$ are observed in films thinner than $15 \mathrm{~nm}^{2}$ The nature and mechanism of polarization switching and polarization relaxation in ultrathin LB films have been attributed to both intrinsic ${ }^{2}$ (bulk) and extrinsic ${ }^{3}$ (nucleation initiated) mechanisms. Recent studies of polarization switching in PVDF by piezoresponse force microscopy (PFM) have demonstrated that switching occurs on a grain by grain basis, with correspondingly high activation energies and broad distributions of activation times. ${ }^{4,5}$ Local switching time studies that average over $100 \mathrm{~s}$ of crystal grains in ferroelectric copolymer LB films support an extrinsic mechanism. ${ }^{6}$ Despite this progress, little is known on the local relaxation dynamics of individual crystals or domains in PVDF.

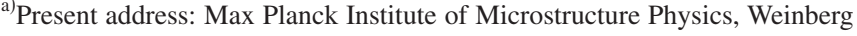
2, D-06120 Halle, Germany. Electronic mail: brodrig@mpi-halle.mpg.de. ${ }^{b)}$ Author to whom correspondence should be addressed. Electronic mail: sergei2@ornl.gov.
}

Here, we report a study of the spatial variability of relaxation behavior in PVDF nanomesas ${ }^{7}$ using time-resolved piezoresponse force spectroscopy (TR-PFS). The thin ferroelectric polyvinylidine fluoride and trifluoroethylene $[\mathrm{P}(\mathrm{VDF}-\mathrm{TrFE})]$ copolymer films were fabricated on oxidized $\left(2-3 \mathrm{~nm}\right.$ native oxide) highly doped ( $n$-type, $\left.\sim 10^{18} / \mathrm{cm}^{3}\right)$, conductive $(\sim 2 \Omega \mathrm{cm}) \quad \mathrm{Si}(100)$ substrates by a LB technique. ${ }^{8}$ Ohmic aluminum contacts were previously formed on the bottom of the wafer by evaporation followed by annealing at $200{ }^{\circ} \mathrm{C}$ for $10 \mathrm{~h}$. The role of the oxide has been addressed in Refs. 9 and 10. De-ionized water with a resistivity of $18 \mathrm{M} \Omega$ and temperature of $(25 \pm 1){ }^{\circ} \mathrm{C}$ was used as the subphase in a clean Teflon ${ }^{\mathrm{TM}}$ Langmuir trough with a total area of $1520 \mathrm{~cm}^{2}$. Then, a solution of vinylidene fluoride (70\%) with trifluoroethylene (30\%) copolymer, $\mathrm{P}(\mathrm{VDF}-\mathrm{TrFE} 70: 30)$, in dimethyl sulfoxide (0.01 wt \% concentration) was dispersed on the top of the water subphase with the aid of an electric pipette and microscope slides. The dispersed copolymer solution became a thin film on the water subphase, and the film was compressed at a rate of $20-60 \mathrm{~cm}^{2} / \mathrm{min}$ by two barriers from the outside toward the center of the trough to reach a target surface pressure of $5 \mathrm{mN} / \mathrm{m}$. Once the target pressure was reached, the film on the water surface was transferred to the Si substrate by horizontal dipping, while keeping the surface pressure constant at $5 \mathrm{mN} / \mathrm{m}$. Prior studies showed that this technique produces films with an average thickness of $1.78 \pm 0.07 \mathrm{~nm}$ per nominal monolayer. ${ }^{11}$ The sample was then annealed in air for $2 \mathrm{~h}$ at $135^{\circ} \mathrm{C}$, with heating and cooling rates of $1{ }^{\circ} \mathrm{C} \mathrm{min}^{-1}$ to form P(VDF-TrFE) nanomesa structures. ${ }^{9}$ The effect of annealing conditions on nanomesa formation has been discussed elsewhere. ${ }^{10}$

PFM imaging and spectroscopy were implemented on a commercial atomic force microscope system (Veeco MultiMode with a Nanonis controller) equipped with an external 


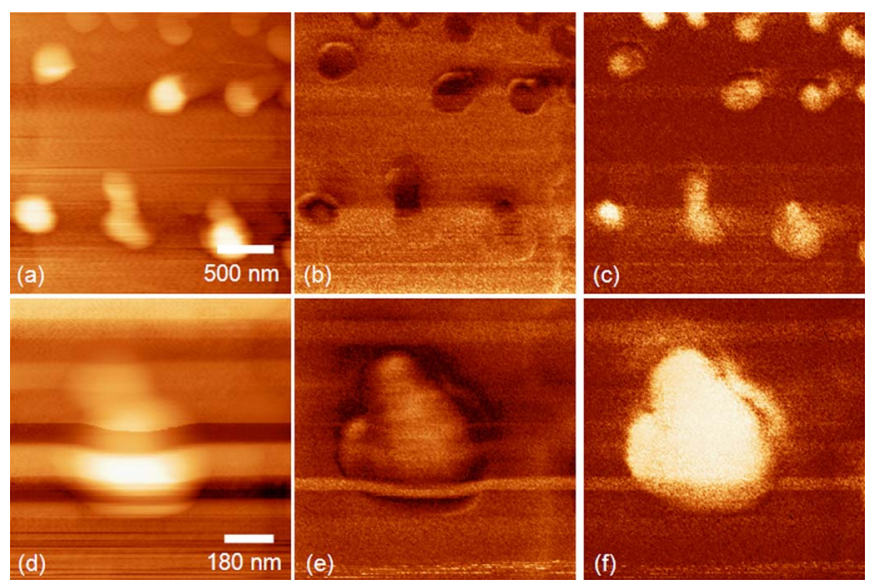

FIG. 1. (Color online) [(a) and (d)] Topography, [(b) and (e)] PFM amplitude, and [(c) and (f)] PFM phase images of PVDF nanomesa structures.

signal generation and data acquisition system similar to that described in Ref. 12. To minimize surface damage, PFM measurements were performed using ultrasoft $\mathrm{Au}-\mathrm{Cr}$ coated Si tips (Micromasch, spring constant $k \sim 0.03 \mathrm{~N} / \mathrm{m}$ ). Imaging was performed using a $1.1 \mathrm{MHz}, 5 \mathrm{~V}$ bias. As the aluminum contact on the back side of the doped Si substrate was used as the back side electrical contact in the PFM measurements, a voltage drop of no more than $1 \mathrm{~V}$ is expected to occur across the thin oxidized layer (as estimated for flat geometry; for a localized PFM tip, the field concentration in PVDF should be even larger). ${ }^{13}$

Topography, PFM amplitude, and PFM phase images of PVDF nanomesas are shown in Figs. 1(a)-1(c), respectively.
The images demonstrate strong electromechanical contrast at the nanomesas, as expected for the ferroelectric state of the polymer. The PFM phase image indicates that mesas are in a uniformly polarized state.

To probe the polarization relaxation at a single point, the microscope was configured in the TR-PFS mode, as illustrated in Figs. 2(a) and 2(b). A setting pulse of $10 \mathrm{~V}$ is applied to the probe for $20 \mathrm{~ms}$, and then the bias is turned off for the following $200 \mathrm{~ms}$. The evolution of the electromechanical response during this relaxation stage is measured using a $1.1 \mathrm{MHz}$ ac signal. The sequence is repeated five times, and the results are averaged, resulting in a $1.1 \mathrm{pixel} / \mathrm{s}$ data acquisition rate. Several relaxation curves (smoothed by adjacent averaging) from the substrate and nanomesa are shown in Figs. 2(c) and 2(d), respectively. The curves are approximated using the exponential decay,

$$
\mathrm{PR}=A_{1} \exp ^{(-t / \tau)}+B
$$

where PR is the piezoresponse signal, $A_{1}$ is the relaxation amplitude (switchable response), $\tau$ is the time constant, and $B$ is the nonrelaxing component (remanent response). In automatic data analysis, the fitting is performed starting from delay time, $t=t_{0}$. Note that relatively high noise levels corresponding to small $(\sim 10 \mathrm{pm} / \mathrm{V})$ piezoelectric coefficients of PVDF and limited pixel times do not allow elucidation of the exact relaxation kinetics (e.g., distinguishing exponential and stretched exponential responses). Relaxation behavior in $\mathrm{P}(\mathrm{VDF}-\mathrm{TrFE})$ nanomesas could also arise from charge on the surface; however, this response is anticipated to appear as a uniform offset on the images and cannot account for the
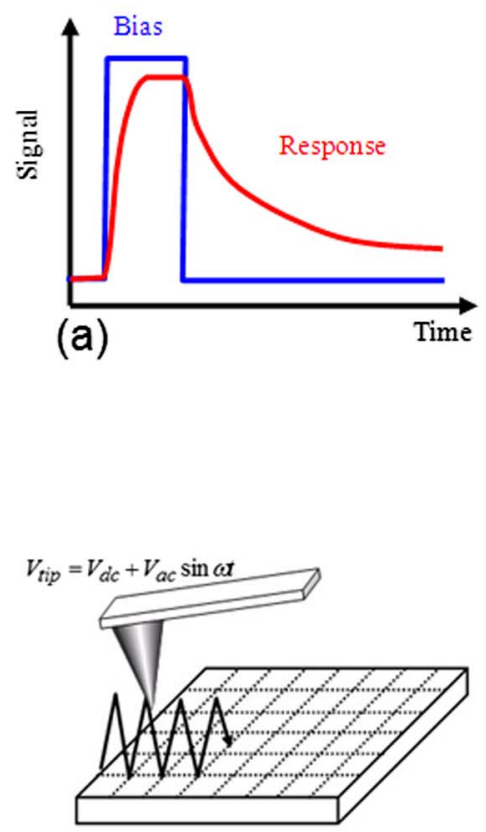

(b)
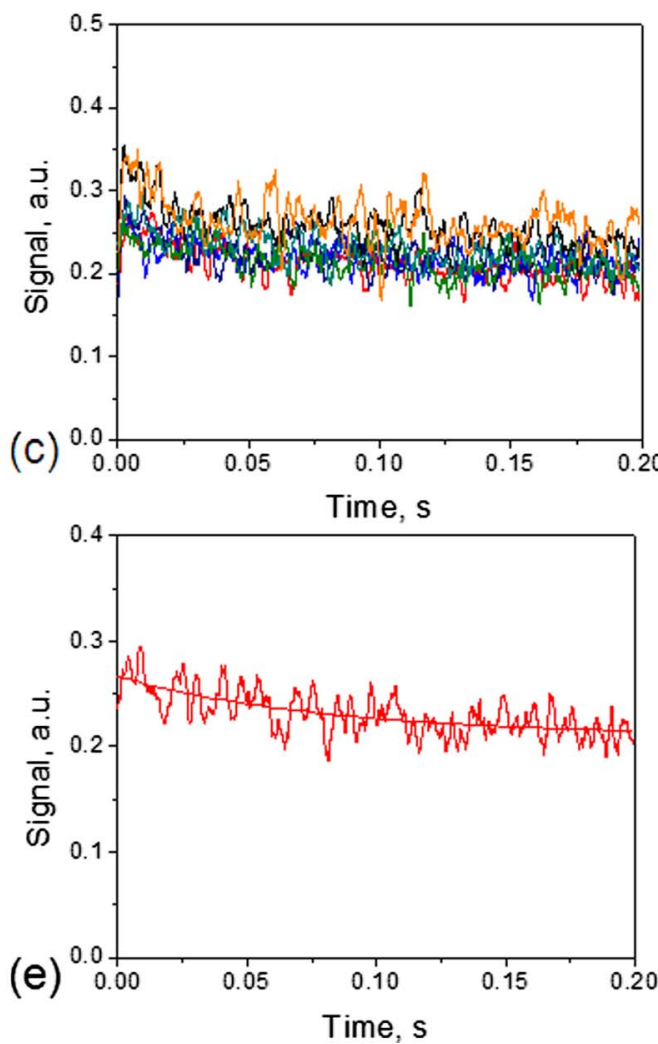
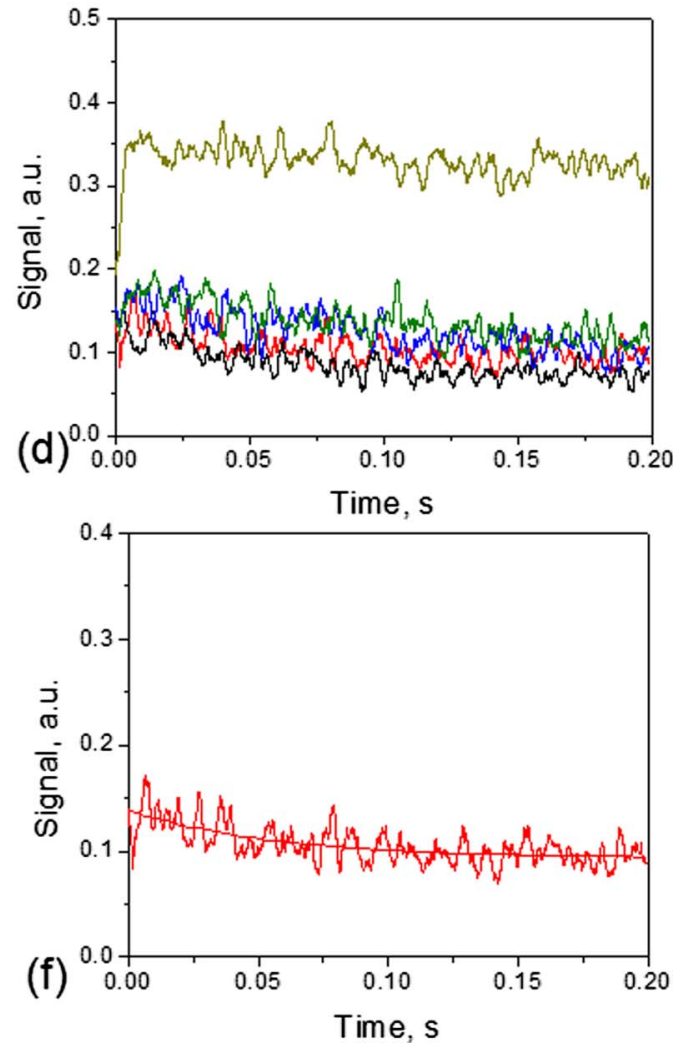

FIG. 2. (Color online) [(a) and (b)] Schematics of TR-PFM. Smoothed (54 point adjacent averaging) relaxation curves from (c) the substrate and (d) PVDF nanomesas. Relaxation curves from (e) the substrate and (f) a PVDF nanomesa, and corresponding fits. 


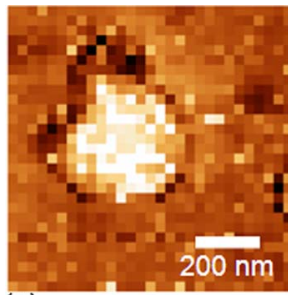

(a)

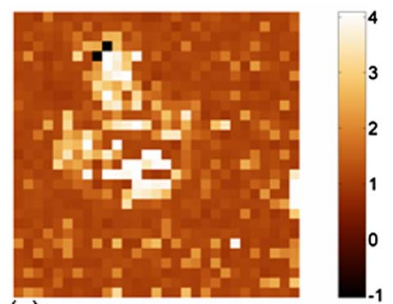

(c)

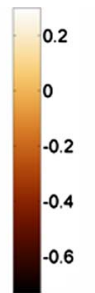

(b)

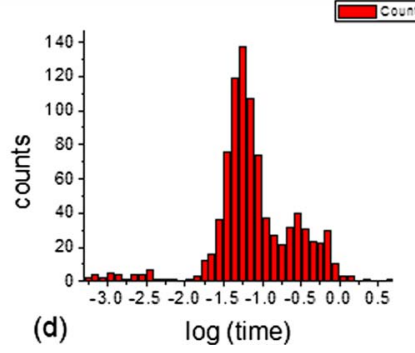

FIG. 3. (Color online) Relaxation images of the (a) remanent amplitude, (b) relaxation amplitude, and (c) relaxation time constant [in seconds]. (d) Histogram of the log of the relaxation time.

sharp variability of relaxation behavior within the nanomesa discussed below.

To probe variability of relaxation behavior on a sample surface, the relaxation measurements are performed over a grid of $N \times N$ points, as illustrated schematically in Fig. 2(b). The resulting three dimensional data array is fitted by Eq. (1) [Figs. 2(e) and 2(f)], and coefficients at each point are plotted as two dimensional maps. Shown in Fig. 3 are the resulting time-resolved spectroscopic maps for PVDF nanomesas $(N=30)$. Figure 3(a) clearly shows a large remanent response within the nanomesa region, suggesting that most of the response is either constant or relaxes slowly on the time scale of the experiment. The relaxation amplitude [Fig. 3(b)] is higher within the mesa, as anticipated. However, nonzero switchable contrast was observed on the (nominally) bare $\mathrm{SiO}_{2}$ surface as well. This behavior can be attributed either due to the presence of residual PVDF contaminants on the surface (or tip) or time-dependent phenomena associated with electrostatic and electrocapillary condensation at the tip-surface junction. $^{14}$

The most interesting contrast is observed in the relaxation time constant image [Fig. 3(c)]. Note that the majority of the nanomesa is characterized by much larger relaxation times $(\sim 0.1-1 \mathrm{~s})$ than the substrate $(0.05 \mathrm{~s})$. Furthermore, the TR-PFS measurements demonstrate an inhomogeneous relaxation time distribution within the nanomesa. Note that the orientation and clustering of the regions with different relaxation times are such that they minimize the probability of topographic cross-talk or tip-induced changes on observed contrast.

To summarize, we have developed a spatially resolved imaging approach, time-resolved piezoresponse spectroscopic imaging, to probe the spatial variability of relaxation behavior in nanoscale ferroelectrics. The approach was applied to study polarization dynamics in $\mathrm{P}(\mathrm{VDF}-\mathrm{TrFE})$ nanomesas. The relaxation times even within a single nanomesa were found to be relatively large, on the scale of $0.1-1 \mathrm{~s}$. The spatially resolved mapping illustrated that relaxation times are inhomogeneous within the nanomesa. While the origins of this behavior are unknown, this behavior can be associated with the presence of an internal grain boundary between two grains in a mesa structure. Overall, we believe that this technique has the potential for nanoscale mapping of relaxation behavior in ferroelectric polymers and relaxors.

The research was supported in part by the Division of Materials Science and Engineering, Basic Energy Sciences, U.S. Department of Energy at Oak Ridge National Laboratory, which is managed by UT-Battelle, LLC. Work at the University of Nebraska was supported by the National Science Foundation and the Nebraska Research Initiative. One of the authors (B.J.R.) acknowledges the support of the Alexander von Humboldt Foundation.

${ }^{1}$ A. J. Lovinger, Science 220, 1115 (1983).

${ }^{2}$ S. Ducharme, V. M. Fridkin, A. V. Bune, S. P. Palto, L. M. Blinov, N. N. Petukhova, and S. G. Yudin, Phys. Rev. Lett. 84, 175 (2000); G. Vizdrik, S. Ducharme, V. M. Fridkin, and S. G. Yudin, Phys. Rev. B 68, 094113 (2003).

${ }^{3}$ H. Kliem and R. Tadros-Morgane, J. Phys. D 38, 1960 (2005).

${ }^{4}$ B. J. Rodriguez, S. Jesse, S. V. Kalinin, J. Kim, S. Ducharme, and V. M. Fridkin, Appl. Phys. Lett. 90, 122904 (2007).

${ }^{5}$ V. S. Bystrov, I. K. Bdikin, D. A. Kiselev, S. G. Yudin, V. M. Fridkin, and A. L. Kholkin, J. Phys. D 40, 4571 (2007).

${ }^{6}$ R. V. Gaynutdinov, O. A. Lysova, A. L. Tolstikhina, S. G. Yudin, V. M. Fridkin, and S. Ducharme, Appl. Phys. Lett. 92, 172902 (2008).

${ }^{7}$ M. Bai and S. Ducharme, Appl. Phys. Lett. 85, 3528 (2004); M. Bai, M. Poulsen, and S. Ducharme, J. Phys.: Condens. Matter 18, 7383 (2006).

${ }^{8}$ A. Sorokin, S. Palto, L. Blinov, V. Fridkin, and S. Yudin, J. Phys.: Condens. Matter 6, 61 (1996).

${ }^{9}$ M. J. Bai and S. Ducharme, Appl. Phys. Lett. 85, 3528 (2004).

${ }^{10}$ M. Bai, M. Poulsen, and S. Ducharme, J. Phys.: Condens. Matter 18, 7383 (2006).

${ }^{11}$ M. Bai, A. V. Sorokin, D. W. Thompson, M. Poulsen, S. Ducharme, C. M. Herzinger, S. Palto, V. M. Fridkin, S. G. Yudin, V. E. Savchenko, and L. K. Gribova, J. Appl. Phys. 95, 3372 (2004).

${ }^{12}$ S. Jesse, H. N. Lee, and S. V. Kalinin, Rev. Sci. Instrum. 77, 073702 (2006).

${ }^{13}$ T. J. Reece, S. Ducharme, A. V. Sorokin, and M. Poulsen, Appl. Phys. Lett. 82, 142 (2003).

${ }^{14}$ B. L. Weeks, M. W. Vaughn, and J. J. De Yoreo, Langmuir 21, 8096 (2005). 